\title{
Effective Graft Preservation by Following a Standard Protocol for the Treatment of Knee Joint Infection after Anterior Cruciate Ligament Reconstruction
}

\author{
Robin Otchwemah, MD ${ }^{1,2}$ Jan-Hendrik Naendrup ${ }^{1}$ Frauke Mattner, MD ${ }^{2}$ Thorsten Tjardes, MD ${ }^{1}$ \\ Holger Bäthis, $\mathrm{MD}^{1}$ Sven Shafizadeh, MD ${ }^{1}$ \\ ${ }^{1}$ Department of Trauma Surgery and Orthopedic Surgery, \\ Cologne-Merheim Medical Center, University of \\ Witten/Herdecke, Köln, Germany \\ 2 Institute for Hygiene, Clinics Cologne, Cologne-Merheim Medical \\ Center, Köln, Germany

\begin{abstract}
Address for correspondence Robin Otchwemah, MD, Department of Trauma Surgery and Orthopedic Surgery, Institute for Hygiene, Cologne-Merheim Medical Center, University of Witten/Herdecke, Ostmerheimerstr. 200, D-51109 Köln, Germany

(e-mail: otchey@gmail.com).
\end{abstract}

J Knee Surg 2019;32:1111-1120.

\begin{abstract}
Knee joint infections constitute a rare but devastating complication after anterior cruciate ligament $(\mathrm{ACL})$ reconstruction. We hypothesized that effective infection therapy and graft preservation is possible following a standard treatment protocol. We retrospectively analyzed all patients admitted to our center with suspected infection of the knee after $A C L$ reconstruction between 2010 and 2012. Following a standardized protocol, blood samples were drawn and synovial fluid was analyzed. Furthermore, the protocol consisted of arthroscopic lavages and debridements of the anterior and posterior joint compartments over three incisions, and targeted antibiotic therapy over a period of 6 weeks. Surgeries were repeated every 2 days until clinical signs of infections resolved, but at least two times. Mean observation period was 10 months. Forty-one patients aged 31 ( \pm 9.9) years and admitted $14( \pm 7.5$ ) days after ACL reconstruction were included. Pathogens were found in 34 patients and

\section{Keywords}

- anterior cruciate ligament reconstruction

- infection

- joint

- arthritis

- staphylococcus coagulase-negative staphylococci were isolated most commonly (31 isolates in 28 patients). Quinolones were the most commonly used antibiotic agents. Mean number of operations was $3.8( \pm 1.4)$. Following the standard protocol, primary successful infection treatment with graft preservation was possible in 37 of the included 41 patients. Graft preservation was achieved in $100 \%$ of the included patients with Gaechter stage 1 and 2 infections. Knee joint infection after ACL reconstruction was successfully treated following a standardized protocol, and graft preservation was reliably achieved especially in cases with infections at an early stage.
\end{abstract}

With an incidence ranging from 0.14 to $0.78 \%{ }^{1,2}$ knee joint infections constitute a rare complication after anterior cruciate ligament $(\mathrm{ACL})$ reconstruction. Despite its infrequent occurrence, postoperative infection is a devastating consequence in this high-demanding patient population, and the primary treatment goal is the protection of graft and cartilage to preserve joint stability and long-term function.

After ACL reconstruction, infections most commonly appear only a few weeks after surgery, which means that it requires a high level of attention to distinguish postoperative received

February 22, 2017

accepted

September 29, 2018

published online

November 26, 2018
Copyright $\odot 2019$ by Thieme Medical Publishers, Inc., 333 Seventh Avenue, New York, NY 10001, USA. Tel: +1(212) 584-4662.
DOI https://doi.org/ 10.1055/s-0038-1675794. ISSN 1538-8506. 
from infectious findings ${ }^{3-5}$ and that this complication frequently delays the important early phase of rehabilitation. ${ }^{6}$ Diagnosis has to be made quickly, and treatment must be as efficient and as little invasive as possible to enable the patient to return to standard rehabilitation protocol immediately.

Although the cornerstones of treatment are widely accepted-arthroscopy or arthrotomy with synovial irrigation and debridement (I\&D) as well as systemic antibioticsthere is still no consensus about detailed, validated treatment algorithms. The length of antibiotic therapy ranges from 5 to 90 days, and depending on clinical findings and biomarker levels, repeated irrigations after 48 to 72 hours are considered. $^{2,7}$ Occasionally, a graft removal is necessary, which increases the treatment duration and may lead to further reconstructive procedures and impaired functional outcome. $^{1}$

We hypothesized that effective infection therapy and reliable graft preservation is possible following a standard protocol consisting of at least three arthroscopic lavages and debridements of the anterior and posterior joint compartments over three incisions, and targeted antibiotic therapy over a period of 6 weeks. Furthermore, we present the clinical and biomarker findings, as well as the bacterial spectrum and clinical outcomes of a large series of patients with knee infections following ACL reconstruction surgery.

\section{Materials and Methods}

We analyzed patients who were referred to our specialized center for joint surgery with symptoms of knee infection after ACL reconstruction between January 2010 and December 2012 in a retrospective cohort study. Patients who were treated for ACL infections were prospectively listed in a table by the head of joint surgery department, and detailed data were collected retrospectively from the electronic hospital information system. The ethics committee of the University Witten/Herdecke approved the study protocol.

Depending on their exact medical history, all patients presenting with increasing pain, increasing swelling, increasing limitations of knee function, and at least one common sign of infection (temperature $>38^{\circ} \mathrm{C}$, local hyperthermia of the knee, shivering, and malaise) after ACL reconstruction were considered as cases of infections and included. Patients were excluded from this study if they had decreasing or stable levels of pain, swelling and limitations of knee function, or no common sign of infection as mentioned previously.

Following a standard protocol ( - Fig. 1), blood samples for the evaluation of C-reactive protein (CRP) levels and leucocyte count were drawn. A diagnostic aspiration of synovial fluid for macroscopic examination and for microbiological testing in an anaerobic and aerobic blood culture bottle (Bactec) was performed. Additionally, plain radiographs of the knee in two planes were taken.

Furthermore, one senior surgeon performed an arthroscopic lavage and debridement of the anterior and posterior joint compartments using an anteromedial, anterolateral, and posteromedial arthroscopic access within 24 hours after admission. No perioperative antibiotic prophylaxis was administered. The procedure was repeated with a 20-day interval between each operation until no arthroscopic signs of infection according to Gaechter's classification ${ }^{8}$ (purulent synovial fluid, infectious affections of the synovialike thickening, reddening, petechial bleedings, fibrin layers) were evident during surgery, but at least two times, which means each patient underwent at least three operative interventions. All patients received nonsteroidal anti-inflammatory drugs (NSAIDs) following a standard protocol (ibuprofen $600 \mathrm{mg}$ every 6 hours, or metamizole $500 \mathrm{mg}$ every 6 hours
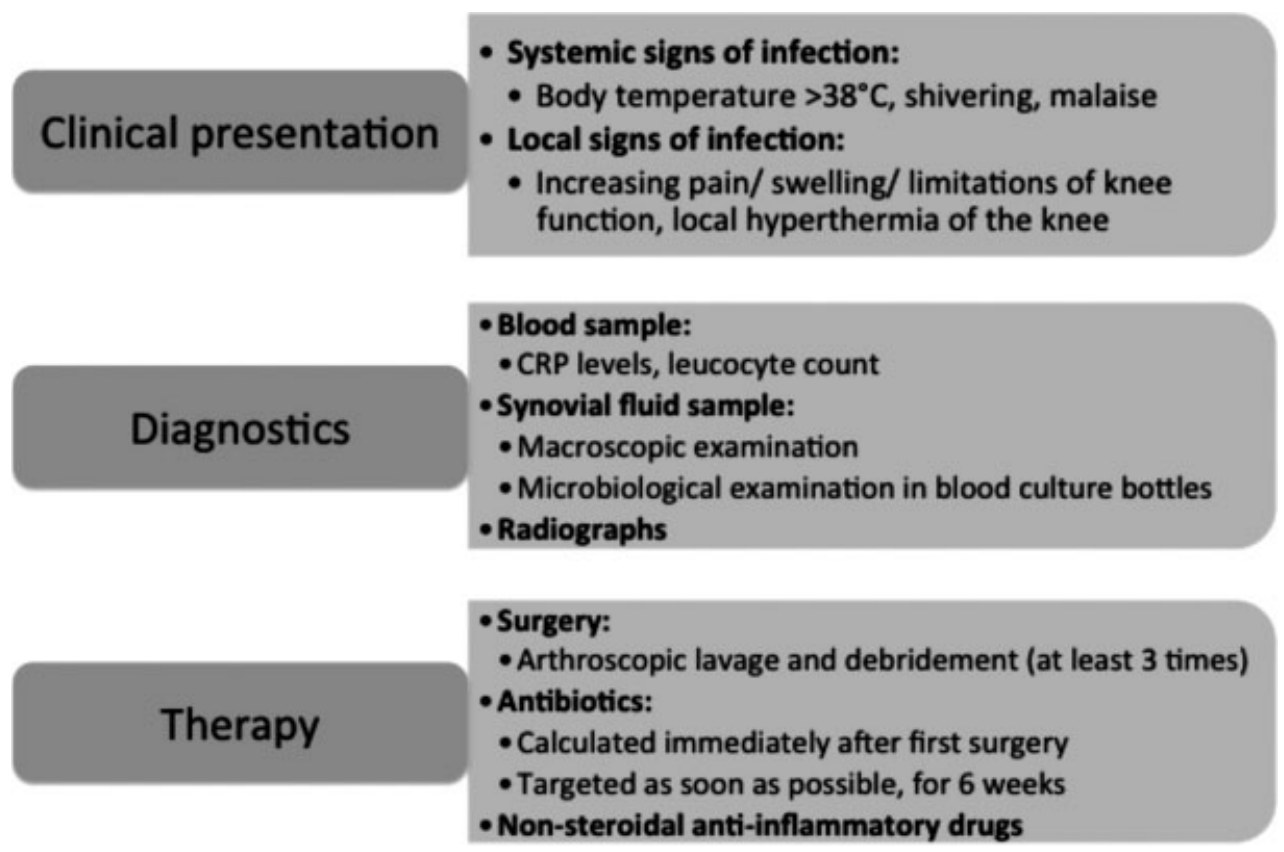

Fig. 1 Clinical pathway: key elements of presentation and diagnostic and therapeutic steps. 
in case of renal disease). Calculated antibiotic therapy with gram-positive coverage in recommended dosage for grampositive bacteria was started immediately after initial surgery. Targeted therapy was administered as soon as microbiological data were available and lasted 6 weeks.

During follow-up treatment, weight load was not applied to the affected knee until the last surgery was completed. Afterward, weight-bearing with $20 \%$ of bodyweight and free range of motion, supported by a continuous passive motion device, was performed. Depending on the decline of inflammation, patients returned to their usual post-ACL follow-up treatment plan as soon as possible. They started the excercise plan, at the stage it was interrupted, when it came to the joint infection.

For bacterial identification and resistance testing, the VITEK 2 System (bioMérieux Inc.) was used and blood cultures were cultivated in the Bactec System (Becton Dickinson). All samples were cultured for 14 days.

For further analysis, medical records and operative reports were retrospectively analyzed. Demographic data and specific surgical details of the index ACL procedure, as well as clinical presentation at the time of first revision surgery, type and onset of symptoms, laboratory parameters (CRP, white blood cell counts), time to arthroscopic I\&D, number of revisions, type of antibiotics used, bacteria detected in microbiological testing, and graft retention or removal were obtained. The Gaechter classification was used to categorize joint damage, ${ }^{8}$ and infections were divided into acute infections if they occurred within the first 2 weeks, subacute infections if they occurred between 2 weeks and 2 months, and late infections if they occurred more than 2 months after ACL reconstruction. Follow-up examination was performed by a senior surgeon who assessed function and knee joint stability using the Lachman and pivot shift tests. Any range of motion with a flexion of less than 140 degrees and without full extension was referred to as "limitation of joint function."

Statistical analysis was performed using Microsoft Excel. Descriptive results are demonstrated as mean and standard deviation. Two-tailed $t$-test was used for the comparison between infections with high- and low-virulent pathogens. The significance level was set to 0.05 .

\section{Results}

\section{Patients}

Forty-one patients (32 males, 9 females) aged $31( \pm 9.9)$ years were included, of which 18 had ACL reconstruction in our department. Two patients were adipose with a body mass index above 30. One was suffering from diabetes mellitus type 2 and one from depression. Another patient was suffering from glomerulonephritis and Hashimotòs thyroiditis and was adipose.

In 38 patients, hamstring autografts were used for ACL reconstruction; three patients received a quadriceps tendon autograft (-Table 1). Fixation was managed using tibial, bioresorbable interference screws and femoral endobuttons. Nineteen patients had additional meniscus surgery during
ACL reconstruction. Ten of those patients had partial meniscectomies. Nine had suture refixations. Mean number of operations for infection treatment was $3.8( \pm 1.4)$, mean length of hospital stay for infection treatment was $13( \pm 2)$ days, and mean observation period after onset of joint infection treatment was $10( \pm 7)$ months (minimum: 6 months; maximum: 31 months).

\section{Clinical Presentation}

All infections were acute or subacute. Average time between the $\mathrm{ACL}$ reconstruction index procedure and the time of readmission was 14 ( \pm 7.5 ) days. At the time of readmission, all patients reported increasing pain, increasing swelling, and increasing limitations of the knee joint function. Thirty-eight patients had local hyperthermia of their knee, and in 38 patients, body temperature was slightly elevated $\left(37-38.3^{\circ} \mathrm{C}\right)$. All patients showed at least one of those two symptoms. Thirty-six of the patients were suffering from malaise (-Table 1). One patient needed to be admitted to the intensive care unit due to septic coagulopathy.

\section{Diagnostics}

Elevated CRP values $(126 \pm 66 \mathrm{mg} / \mathrm{L})$ were present in all cases, whereas only 11 patients showed elevated white blood cell counts $(9.1 \pm 3 / \mathrm{nL})$ in blood samples. Twenty-four patients were classified as Gaechter stage 1, 15 as Gaechter stage 2 , and 2 as stage 3 knee joint infections (-Table $\mathbf{1}$ ). No joint was classified as Gaechter 4. Thus, all patients showed signs of infection during the diagnostic part of arthroscopy and were considered as knee joint infections following the Centers for Disease Control and Prevention definition (clinical symptoms of infection + positive imaging test). ${ }^{9}$

In all but three cases synovial fluid samples could be aspirated. In those three patients, aspiration was not possible due to cannula clotting. Qualitative, macroscopic synovial fluid analysis showed purulent fluid in 35 cases and bloody fluid in 3 cases. Blood culture analysis of the synovial fluid identified bacteria in 34 of 38 patients. Although the standard protocol did not require synovial fluid swabs, samples were taken from 19 patients. Bacteria could only be identified in nine of those cases.

\section{Microbiology}

Forty-one pathogens were isolated among the 41 study patients, whereby 7 patients had an infection with two different bacterial species and in 7 patients no bacteria could be detected. Coagulase-negative staphylococci (COSTs) were found most commonly (-Fig. 2). Thirty-one COST isolates were found in 28 patients. Three patients had an infection with two different COSTs. Twenty-four COST isolates were resistant against all $\beta$-lactam antibiotics, and 21 additionally showed resistances against one to five other antibiotic substance classes. Thus, prevalence of methicillin resistance among the identified COST in our population was $77 \%$ (-Fig. 3). Staphylococcus aureus was isolated in three, gramnegative strains in five, and Propionibacterium acnes in two cases. In eight cases, high-virulent bacteria (S. aureus and gramnegative rods) were detected. Both patients with Gaechter 


\begin{tabular}{|c|c|c|c|c|c|c|c|c|c|c|c|c|c|c|c|c|c|c|c|c|c|c|c|c|}
\hline 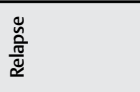 & 1 & 1 & + & I & I & 1 & , & । & , & I & + & 1 & 1 & 1 & + & 1 & 1 & ' & 1 & 1 & 1 & 1 & 1 & I \\
\hline 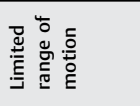 & I & I & I & I & I & I & 1 & I & , & I & 1 & 1 & . & 1 & 1 & 1 & 1 & 1 & 1 & 1 & ' & ' & ' & I \\
\hline 旁衰 & + & + & + & + & 1 & + & + & + & + & + & + & + & + & + & + & , & + & + & + & + & + & + & 1 & + \\
\hline 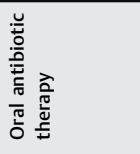 & 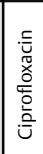 & 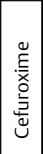 & 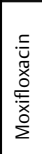 & 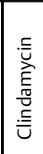 & 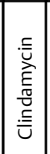 & 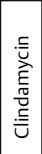 & 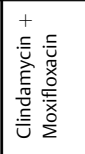 & 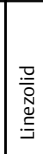 & 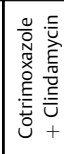 & 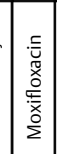 & 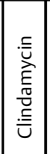 & 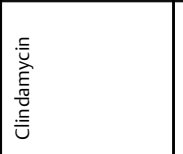 & 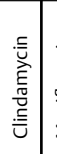 & 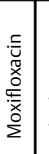 & 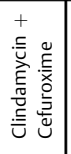 & 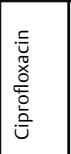 & 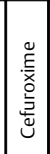 & 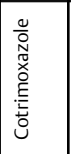 & 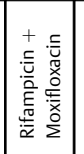 & 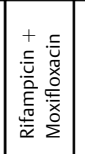 & 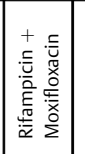 & 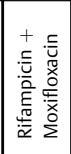 & 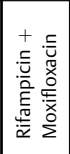 & 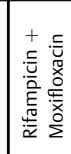 \\
\hline 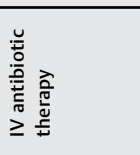 & 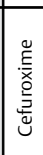 & 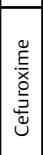 & 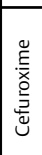 & 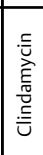 & 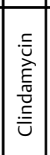 & 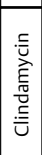 & 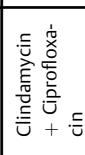 & 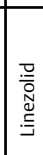 & 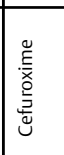 & 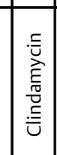 & 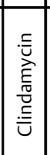 & 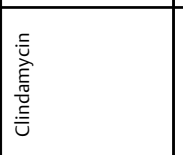 & 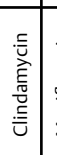 & 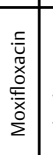 & 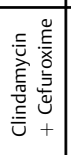 & 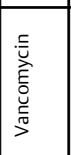 & 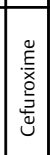 & 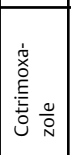 & 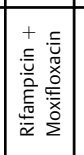 & 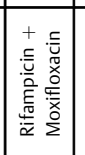 & 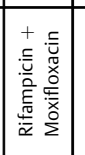 & 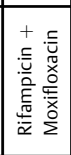 & 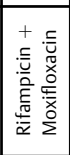 & 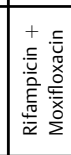 \\
\hline 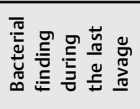 & 1 & 1 & + & ' & 1 & 1 & 1 & I & 1 & I & 1 & 1 & 1 & 1 & 1 & + & 1 & I & 1 & 1 & , & , & 1 & 1 \\
\hline 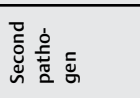 & 䓂 & \begin{tabular}{|l|}
$\frac{2}{2}$ \\
$\frac{5}{2}$
\end{tabular} & \begin{tabular}{|l|}
\multicolumn{2}{c}{} \\
言 \\
ज.
\end{tabular} & 产 & \begin{tabular}{|l|}
$\frac{g}{5}$ \\
$\stackrel{2}{2}$
\end{tabular} & \begin{tabular}{|l|}
$\frac{0}{2}$ \\
$\frac{0}{2}$
\end{tabular} & 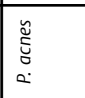 & 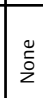 & \begin{tabular}{|l}
$\frac{0}{5}$ \\
$\stackrel{0}{2}$
\end{tabular} & \begin{tabular}{|l|}
$\frac{0}{2}$ \\
$\frac{5}{2}$
\end{tabular} & \begin{tabular}{|l|}
$\frac{0}{0}$ \\
$\stackrel{5}{2}$
\end{tabular} & \begin{tabular}{|l}
$\frac{0}{2}$ \\
$\stackrel{5}{2}$
\end{tabular} & \begin{tabular}{|l|}
$\frac{2}{2}$ \\
$\frac{0}{2}$ \\
\end{tabular} & $\begin{array}{l}\frac{0}{5} \\
\frac{0}{2}\end{array}$ & 产 & 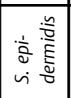 & \begin{tabular}{|l|}
$\frac{2}{2}$ \\
$\frac{2}{2}$
\end{tabular} & 䓂 & 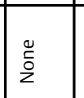 & 㝘 & 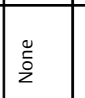 & \begin{tabular}{|l}
$\frac{\eta}{5}$ \\
$\frac{\sigma}{2}$
\end{tabular} & 总 & $\frac{o}{2}$ \\
\hline 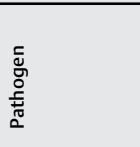 & 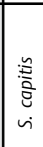 & 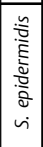 & 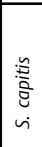 & 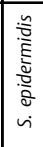 & 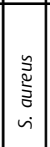 & 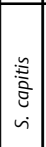 & 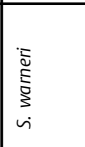 & 营 & 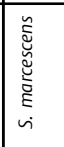 & 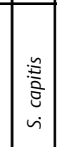 & 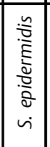 & 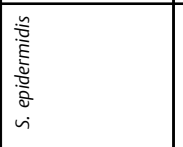 & 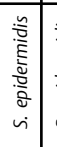 & 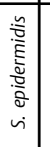 & & 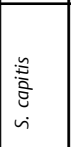 & 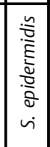 & 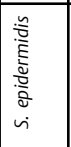 & 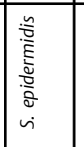 & 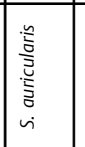 & 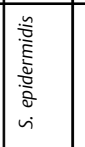 & 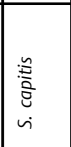 & 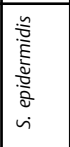 & 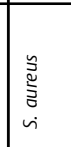 \\
\hline 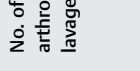 & $m$ & $m$ & 0 & $m$ & in & + & o & $m$ & m & $m$ & $m$ & m & $m$ & 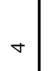 & m & in & $m$ & ナ & n & m & m & 0 & $\infty$ & m \\
\hline 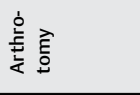 & 1 & 1 & 1 & 1 & 1 & 1 & 1 & ' & 1 & 1 & 1 & 1 & 1 & 1 & 1 & 1 & 1 & 1 & 1 & 1 & 1 & 1 & 1 & I \\
\hline 竧 & - & - & $\sim$ & - & $\sim$ & $\sim$ & $\sim$ & - & $\sim$ & $\sim$ & $\sim$ & - & -1 & $\sim$ & - & $\sim$ & - & - & - & $\sim$ & - & - & $\sim$ & $\sim$ \\
\hline 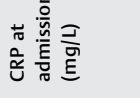 & 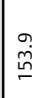 & के & $\begin{array}{l}\tilde{U} \\
\tilde{\rho} \\
\tilde{r}\end{array}$ & $\stackrel{ \pm}{=}$ & $\mid \begin{array}{l}\tilde{f} \\
\stackrel{\sim}{\sim} \\
\sim\end{array}$ & î. & $\stackrel{\substack{\infty \\
\stackrel{\rho}{\underline{j}}}}{ }$ & 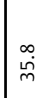 & 总 & ڤ் & $\mid \begin{array}{l}0 \\
\stackrel{0}{0} \\
\stackrel{1}{-}\end{array}$ & 审 & \begin{tabular}{|l|} 
\\
$\stackrel{0}{6}$ \\
$\stackrel{0}{0}$
\end{tabular} & $\stackrel{\infty}{\rightleftharpoons}$ & $\overline{\tilde{v}}$ & $\begin{array}{l}\stackrel{\infty}{\circ} \\
\stackrel{\leftrightarrow}{2}\end{array}$ & 兽 & 畒 & $\underset{\infty}{+}$ & $\begin{array}{c}\bar{\infty} \\
\infty \\
\infty\end{array}$ & $\stackrel{\mathcal{N}}{\simeq}$ & 骂 & {$\left[\begin{array}{c}\infty \\
\infty \\
\infty \\
-\infty\end{array}\right.$} & $\stackrel{\circ}{\stackrel{9}{ }}$ \\
\hline$\frac{\frac{\pi}{0}}{\frac{\pi}{\pi}}$ & 1 & + & + & + & + & + & + & I & + & + & + & + & + & + & + & 1 & + & + & + & + & + & + & + & + \\
\hline 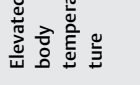 & I & + & + & + & + & + & . & I & + & + & + & + & + & + & + & + & + & + & + & + & + & + & + & + \\
\hline 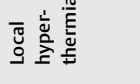 & + & + & + & + & + & + & + & + & + & + & + & + & + & + & + & + & 1 & + & + & + & I & + & 1 & + \\
\hline 胥 & 1 & 1 & + & I & + & 1 & + & I & + & + & 1 & 1 & 1 & 1 & 1 & + & 1 & + & 1 & 1 & ' & + & 1 & 1 \\
\hline 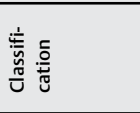 & 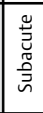 & 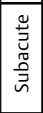 & 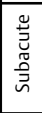 & 荌 & 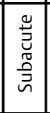 & 苞 & 总 & 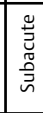 & 茕 & 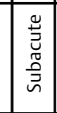 & 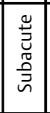 & 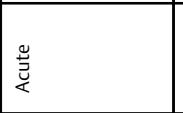 & 旁. & 莣 & 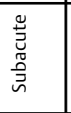 & 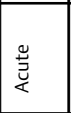 & 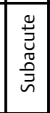 & 蒙 & 蒿 & 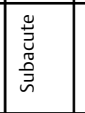 & 苛 & 萤 & 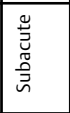 & 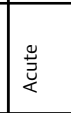 \\
\hline 崖蒙 & $I$ & $I$ & $I$ & $I$ & $I$ & $I$ & 0 & $I$ & 0 & $I$ & \pm & $I$ & \pm & $I$ & I & I & $I$ & $I$ & $I$ & 0 & I & I & $I$ & $I$ \\
\hline $\bar{y}$ & $\begin{array}{l}\frac{0}{5} \\
\frac{0}{2}\end{array}$ & 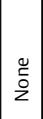 & 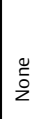 & 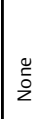 & 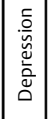 & \begin{tabular}{|l|}
$\frac{a}{2}$ \\
$\stackrel{2}{2}$
\end{tabular} & 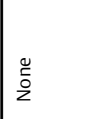 & $\frac{\mathrm{o}}{2}$ & 总 & 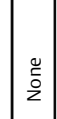 & \begin{tabular}{|l|} 
\\
$\frac{0}{2}$ \\
$\frac{5}{2}$
\end{tabular} & 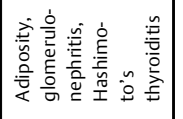 & 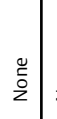 & \begin{tabular}{|l|}
\multirow{2}{0}{} \\
$\frac{\hat{o}}{2}$
\end{tabular} & 产 & 蒿 & $\begin{array}{l}\text { 蒿 } \\
\text { 产 } \\
\frac{1}{4}\end{array}$ & 蒿 & 䓂 & 膏 & 䓂 & 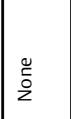 & $\begin{array}{l}\text { 商 } \\
\text { 高 } \\
\text { 妾 }\end{array}$ & 蒿 \\
\hline 速る & i & F & $\stackrel{\sim}{\sim}$ & $m$ & $\infty$ & 2 & $\bar{N}$ & $\stackrel{\infty}{\longrightarrow}$ & 核 & in & $\approx$ & $\stackrel{m}{m}$ & 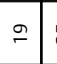 & $\stackrel{\sim}{\sim}$ & $\stackrel{\infty}{\leftarrow}$ & ని & q & $\AA$ & $\stackrel{\infty}{\leftarrow}$ & $\stackrel{\text { I }}{ }$ & F & \& & I & $\approx$ \\
\hline 言 & - & $\sim$ & $m$ & o & in & 0 & $\wedge$ & $\infty$ & $a$ & $\circ$ & $=$ & $\simeq$ & $m$ & \pm & 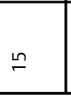 & $\stackrel{\circ}{\circ}$ & $=$ & $\stackrel{\infty}{-}$ & $\stackrel{9}{2}$ & i & $\bar{N}$ & $\approx$ & $\approx$ & 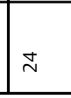 \\
\hline
\end{tabular}




\begin{tabular}{|c|c|c|c|c|c|c|c|c|c|c|c|c|c|c|c|c|c|}
\hline 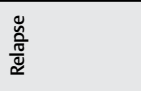 & 1 & 1 & I & I & 1 & I & ' & 1 & 1 & I & 1 & 1 & 1 & 1 & 1 & 1 & 1 \\
\hline 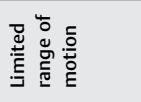 & 1 & 1 & I & ' & I & I & 1 & 1 & 1 & 1 & + & 1 & 1 & 1 & 1 & 1 & I \\
\hline 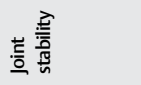 & + & + & + & + & + & + & + & + & + & + & + & + & + & + & + & + & + \\
\hline 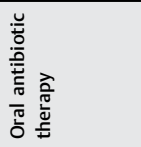 & 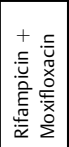 & 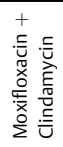 & 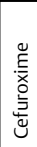 & 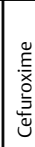 & 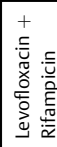 & 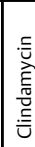 & 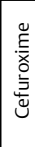 & 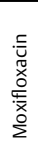 & 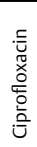 & 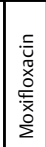 & 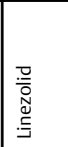 & 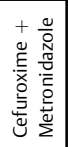 & 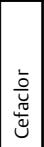 & 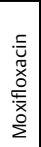 & 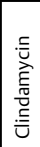 & 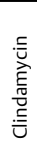 & 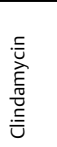 \\
\hline 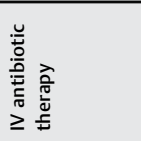 & 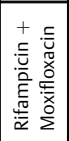 & 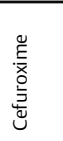 & 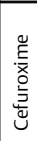 & 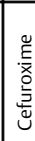 & 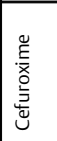 & 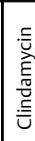 & 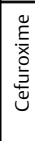 & 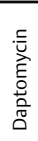 & 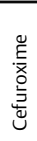 & 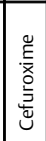 & 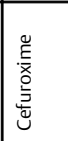 & 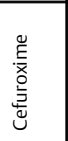 & 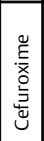 & 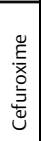 & 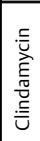 & 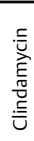 & 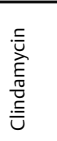 \\
\hline 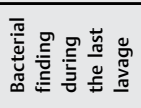 & I & 1 & 1 & I & I & I & 1 & 1 & 1 & I & 1 & 1 & I & 1 & 1 & 1 & 1 \\
\hline 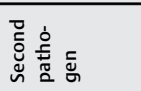 & 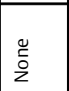 & $\frac{\tilde{0}}{2}$ & $\begin{array}{l}\stackrel{0}{\tilde{o}} \\
2\end{array}$ & 䓂 & $\frac{\tilde{0}}{2}$ & $\begin{array}{l}0 \\
\frac{0}{2}\end{array}$ & $\begin{array}{l}\tilde{0} \\
\frac{0}{2}\end{array}$ & 蒿 & $\begin{array}{l}\text { 气̆ } \\
\stackrel{0}{2}\end{array}$ & 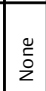 & 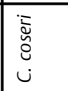 & 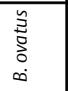 & $\begin{array}{l}\stackrel{0}{\tilde{c}} \\
\stackrel{2}{2}\end{array}$ & $\begin{array}{l}\text { \%̆ } \\
\stackrel{\circ}{2}\end{array}$ & 䓂 & 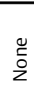 & ن \\
\hline 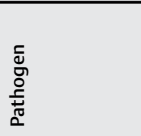 & $\begin{array}{l}\check{y} \\
\tilde{\Xi} \\
\Sigma\end{array}$ & 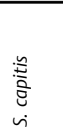 & $\begin{array}{l}\text { : } \\
\text { : } \\
\text { ज. }\end{array}$ & 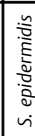 & $\begin{array}{l}n \\
\vdots \bar{z} \\
\bar{\sigma} \\
\text { vi }\end{array}$ & 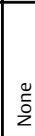 & نे & 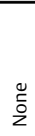 & 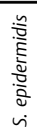 & 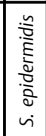 & 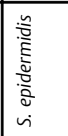 & 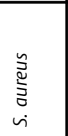 & $\begin{array}{l}n \\
\vdots \bar{z} \\
\vdots \\
\text { vi }\end{array}$ & $\begin{array}{l}. \\
\stackrel{0}{2}\end{array}$ & 气 & 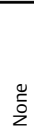 & 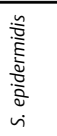 \\
\hline 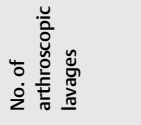 & $m$ & $m$ & $m$ & $m$ & $\nabla$ & $\nabla$ & $\nabla$ & $\sigma$ & $m$ & $m$ & $a$ & $\checkmark$ & $\nabla$ & $m$ & $m$ & $m$ & $m$ \\
\hline 家令 & 1 & 1 & 1 & I & I & I & 1 & 1 & 1 & I & + & 1 & I & 1 & 1 & 1 & 1 \\
\hline 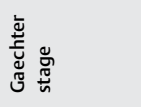 & - & $\sim$ & $\sim$ & - & - & - & - & $\sim$ & $\sim$ & - & $m$ & - & - & - & - & - & - \\
\hline 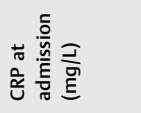 & $\stackrel{\circ}{\stackrel{\infty}{N}}$ & $\begin{array}{l}\stackrel{\sim}{~} \\
\stackrel{m}{\sim}\end{array}$ & ֻั. & $\underset{m}{m}$ & 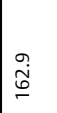 & $\begin{array}{l}\stackrel{0}{\dot{d}} \\
\stackrel{\sim}{1}\end{array}$ & $\underset{\tilde{\nu}}{\tilde{\nu}}$ & $\begin{array}{l}\stackrel{n}{0} \\
\stackrel{\varphi}{\complement}\end{array}$ & $\begin{array}{l}m \\
\dot{f} \\
\dot{m}\end{array}$ & $\stackrel{\text { n̊ }}{\sim}$ & $\stackrel{\infty}{\stackrel{\infty}{\wedge}}$ & $\stackrel{m}{\rightleftarrows}$ & $\hat{i}$ & ¿ & 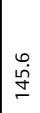 & $\begin{array}{l}\stackrel{0}{\dot{0}} \\
\dot{\infty}\end{array}$ & $\begin{array}{l}\infty \\
\dot{\Xi} \\
\dot{\Xi}\end{array}$ \\
\hline$\frac{\frac{\pi}{n}}{\frac{\pi}{\pi}}$ & + & + & + & + & + & + & + & + & 1 & + & + & 1 & + & + & + & + & + \\
\hline 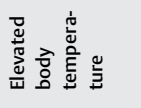 & + & + & + & + & + & + & + & + & + & + & + & + & + & + & + & + & + \\
\hline 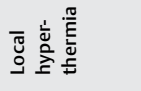 & + & + & + & + & + & + & + & + & 1 & + & + & + & + & + & + & + & + \\
\hline 胥 & I & 1 & I & I & I & I & I & 1 & I & 1 & 1 & 1 & I & + & I & + & 1 \\
\hline 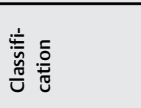 & 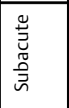 & 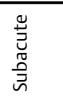 & 莣 & 苞 & 苞 & 芴 & 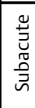 & 苞 & 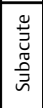 & 苞 & 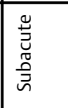 & 苞 & 莣 & 莣 & 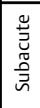 & 莣 & 苞 \\
\hline 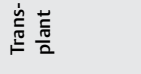 & 포 & I & $I$ & $I$ & $I$ & $I$ & $I$ & $I$ & $I$ & $I$ & $I$ & $I$ & $I$ & $I$ & $I$ & $I$ & $I$ \\
\hline $\bar{z}$ & 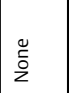 & $\begin{array}{l}\stackrel{0}{\circ} \\
\stackrel{0}{2}\end{array}$ & $\frac{0}{\tilde{0}}$ & 蒿 & 总 & 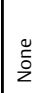 & 蒿 & 产 & $\begin{array}{l}\frac{0}{0} \\
\frac{5}{2}\end{array}$ & 产 & 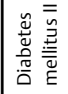 & 蒿 & $\frac{\circ}{2}$ & $\begin{array}{l}\text { 总 } \\
\stackrel{2}{2}\end{array}$ & $\begin{array}{l}\frac{0}{2} \\
\frac{0}{2}\end{array}$ & 总 & 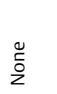 \\
\hline gै & $\stackrel{\infty}{\sim}$ & $\bar{\tau}$ & $\stackrel{m}{m}$ & $m$ & $\stackrel{\infty}{\simeq}$ & F & ని & i & $\stackrel{\infty}{\sim}$ & ণ & 8 & $=$ & in & $\bar{\sim}$ & $\hat{m}$ & $=$ & g \\
\hline 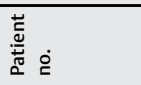 & $\stackrel{\mathscr{N}}{\sim}$ & $\stackrel{\sim}{\sim}$ & $\grave{\sim}$ & $\stackrel{\infty}{\sim}$ & ని & $\stackrel{m}{m}$ & $\bar{m}$ & $\tilde{m}$ & $\stackrel{m}{m}$ & $\stackrel{\vec{m}}{\mathrm{~m}}$ & $\stackrel{\sim}{m}$ & $\stackrel{m}{m}$ & $\hat{m}$ & $\stackrel{\infty}{m}$ & m & 아 & $\bar{\gamma}$ \\
\hline
\end{tabular}




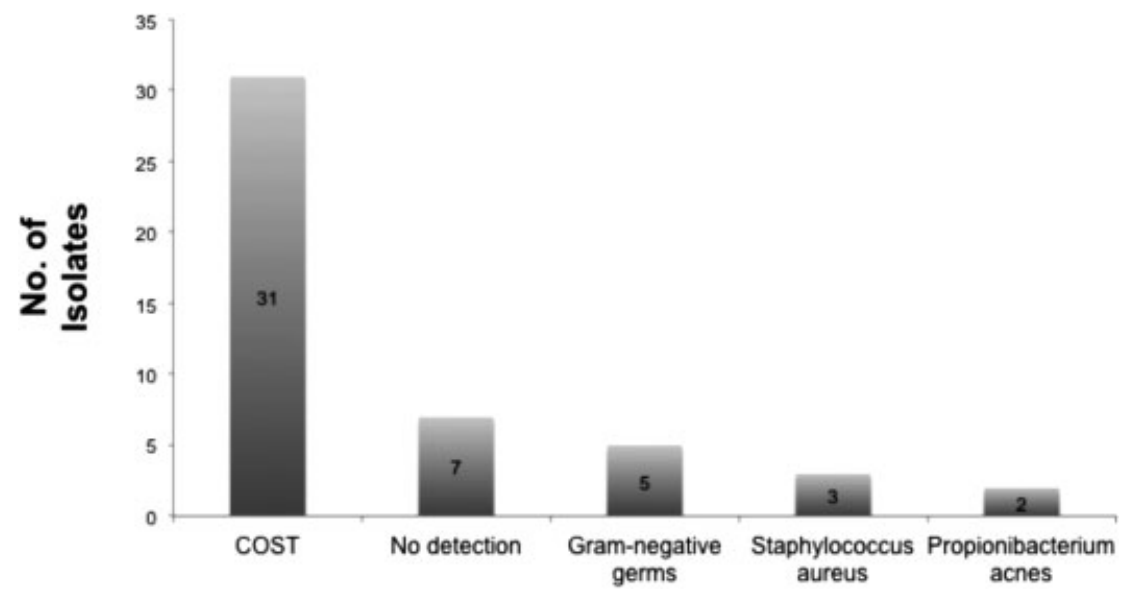

Fig. 2 Pathogens: pathogens and number of affected patients among isolates.

stage 3 showed infections with highly virulent bacteria. One was infected with S. aureus and one with Citrobacter koseri and Staphylococcus epidermidis. There was no significant difference between high- and low-virulent pathogens for the number of surgeries (high virulent: $4.4 \pm 2.1$; low virulent: $3.6 \pm 1.1$; $p=0.19$ ) and the time between ACL replacement and first revision surgery (high virulent: 14 days \pm 6.3 ; low virulent 11 : days $\pm 8.5 ; p=0.37$ ).

\section{Antibiotics}

Quinolones were the most commonly used antibiotics. They were administered in 28 cases during calculated and targeted therapy (-Figs. 4 and 5). Thirteen patients received a combination of two different antibiotics. Quinolones and rifampicin were the most commonly used combination.

\section{Outcome and Graft Preservation}

Completing the aforementioned standard therapy protocol, knee joint infection could be treated successfully in 37 of
41 patients. In these cases, no recurrence of infection was observed, the graft was preserved, and only arthroscopic surgical treatment was performed. However, minor limitations of the knee joint function in one of these cases and anterior joint instabilities in four cases were observed.

In two patients, the knee joint infection relapsed. One patient was readmitted 5 weeks and the other 3 weeks after hospital discharge of the first stay for knee joint infection treatment. Both cases had shown a subacute infection with COST detection. During the relapse, a COST was identified again in one of the patients. No bacteria were found in the other case. Those patients were treated with the same pattern described previously again and achieved full range of motion and joint stability after 6 and 24 months, respectively. One patient with Gaechter stage 3 needed an arthrotomy due to deep infection of the synovia and the joint capsule by $S$. aureus. This 39-year-old patient was suffering from depression. Noncompliance was not suspected.

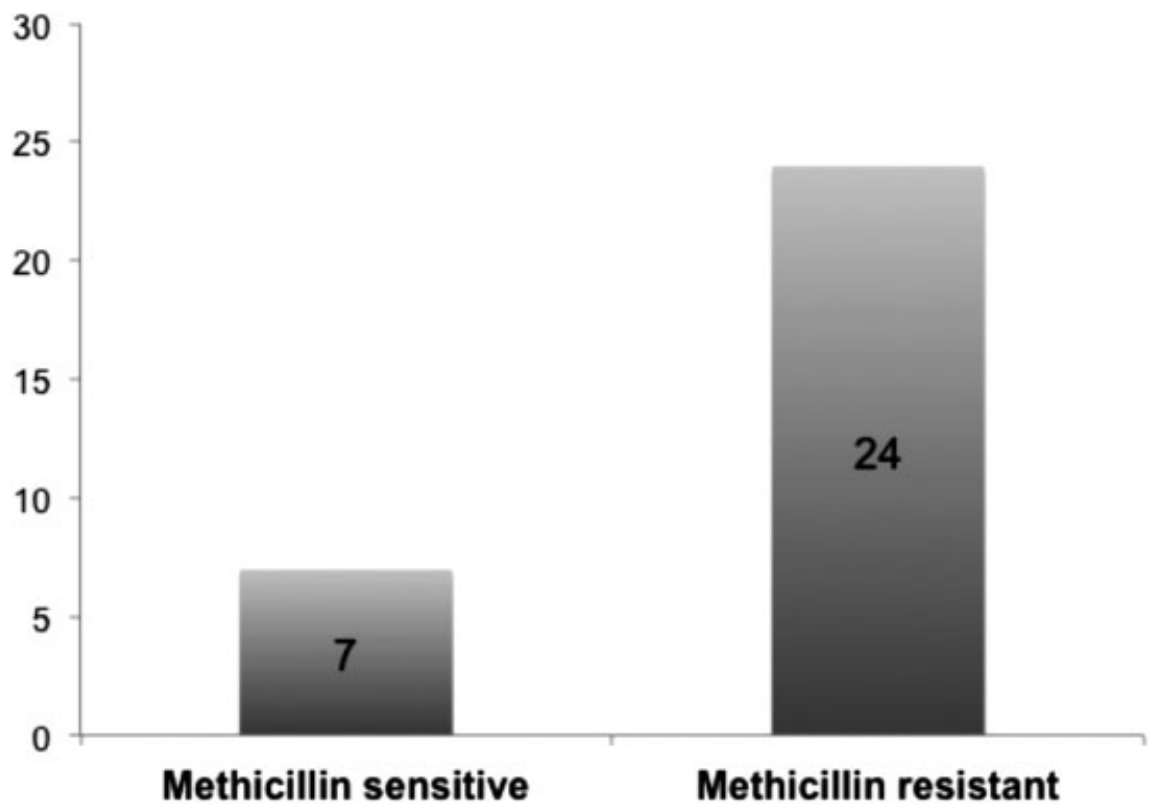

Fig. 3 Frequency of methicillin resistance among patients with staphylococcal infections. 


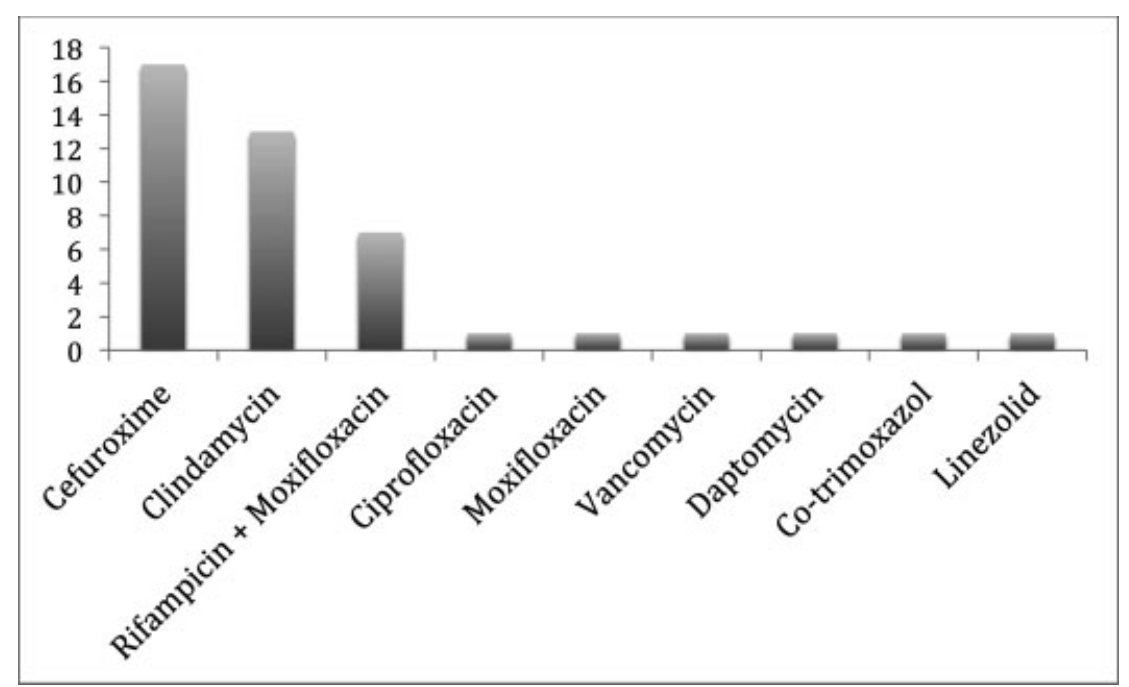

Fig. 4 Calculated antibiotic therapy: number of patients and calculated antibiotic therapy.

Removal of the ACL graft and fixation hardware was necessary in only one case. The patient was suffering from diabetes mellitus and Gaechter stage 3 arthritis. The autografts and hardware could be preserved in all other cases.

No further clinical complications due to surgery or antibiotic therapy were detected.

\section{Discussion}

Following our standard protocol consisting of at least three subsequent arthroscopic I\&D and 6 weeks of targeted antibiotic therapy, primarily successful infection treatment with graft preservation was possible in 37 of the included 41 patients. One patient needed an arthrotomy, and two patients were repeatedly treated with arthroscopic I\&D and antibiotics because of an infectious relapse. In one case, the graft had to be removed. This patient was suffering from diabetes mellitus and Gaechter stage 3 arthritis, whereas in all but one of the other patients, a Gaechter stage
1 or 2 arthritis was diagnosed. Thus, we achieved graft preservation in $100 \%$ of Gaechter stage 1 and 2 arthritis. The applied treatment algorithm provides an easy-toaccomplish, standardized treatment protocol, allowing patients to return to the standard ACL reconstruction rehabilitation process and achieving satisfactory results concerning graft preservation, as well as knee joint stability and knee joint function. A recent meta-analysis found a graft preservation rate of $86 \%$ considering the results of 147 patients. ${ }^{10}$ Data of 16 studies were pooled, of which the largest one included 27 patients. No specific information about the severity of joint space infection was given.

In our study population, knee joint infection after ACL reconstructions presented as an acute infection around 2 weeks after ACL reconstruction with local and systemic inflammatory response. These findings correspond with the existent literature, illustrating the occurrence of most knee joint infections during the first two postoperative months after reconstruction. ${ }^{3-5}$ Clinically presenting with

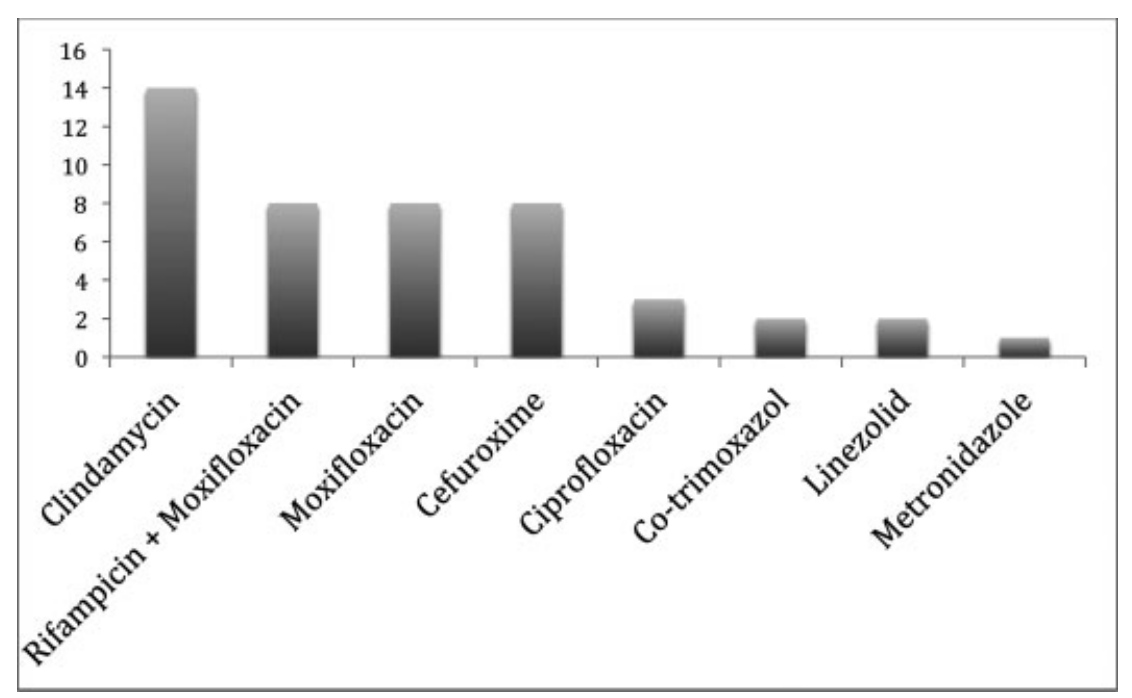

Fig. 5 Targeted antibiotic therapy: number of patients and targeted antibiotic therapy. 
malaise (36 cases), local hyperthermia (38 cases), and elevated body temperature (38 cases) during initial examination, all patients showed symptoms of increasing pain, increasing swelling, and increasing impaired joint function. Regarding the clinical presentation, no differences between the detected pathogens could be recognized. In individual cases, it is particularly difficult to distinguish usual postoperative from infectious findings as fever, malaise, joint pain, reddening, swelling, and hyperthermia are typical and at the same time nonspecific changes after ACL surgery. However, considering our results, those general signs of infection have to lead to further diagnostic steps in the first week after ACL reconstruction.

Beside symptomatic findings, conspicuous synovial fluid after joint tab as well as elevated CRP values were detected in all patients, whereas only 11 had an alteration of white blood cell count. With sensitivity values around $92 \%$ in knee joint infections following ACL reconstruction, ${ }^{6}$ CRP constitutes an important component in diagnostics. The typical course of CRP values in this setting shows an elevation on the first postoperative day, which peaks on day 3 and declines significantly until day 7. It returns to baseline level around day $30,{ }^{11}$ whereas complete normalization time may vary between 2 and 12 weeks interindividually. ${ }^{12}$

A recent study by Wang et al tried to differentiate inflammation from infection using the CRP value. ${ }^{13}$ In their retrospective analysis of 83 patients without and 39 patients with infection after ACL reconstruction, they identified CRP as being more sensitive and specific compared with erythrocyte sedimentation rate. A CRP threshold of $41 \mathrm{mg} / \mathrm{L}$ for septic arthritis showed a sensitivity of $94.1 \%$ and a specificity $97.6 \%$ in the acute postoperative phase. In our patient population, the mean CRP was $126 \pm 66 \mathrm{mg} / \mathrm{L}$, including five cases with CRP levels lower than $41 \mathrm{mg} / \mathrm{L}$ (patients 8, 16, 25, 28, and 31 in - Table 1). In all of those patients, clinical and arthroscopic findings indicated an infection, and in four patients, pathogens were isolated. Either we misjudged normal postoperative changes and blood culture bottles were contaminated, or the threshold might have to be reconsidered.

In the course of this study, we were able to aspirate synovial fluid in 38 of 41 patients. During microbiological diagnostics, which was accomplished in blood culture flasks, pathogens were isolated in 34 of those 38 cases. This high rate of bacterial detections and the method's easy and standardized handling make the bottles a useful diagnostic tool in ACL infections. However, especially in prosthetic joint infections, reliability of synovial fluid culture is controversial as sensitivity ranges between 5 and $92 \% .^{14,15}$ The diagnostic gold standard for septic knee arthritis is joint aspirate analysis including white blood cell count and aerobic and anaerobic cultures. ${ }^{2}$ But different cutoffs for leucocyte levels have been reported in the special setting of early postoperative arthritis. ${ }^{1,2,12}$ At the moment, there is no consensus on a specific number, but during the analysis of five case series, an average number of 82,684 was found in confirmed cases of septic arthritis after ACL reconstruction surgery. ${ }^{2}$ The same group found positive aspirate cultures in $71.4 \%$ of cases during the analysis of nine case series.
Our bacteriological results again highlight the importance of a local surveillance system for the optimization of antibiotic therapy. Staphylococci were the most isolated species in our study, whereby COSTs were more common than $S$. aureus. These findings are generally in line with other publications, although the proportion of $S$. aureus differs between the cohorts. ${ }^{1,16-18}$ The prevalence of COSTs was $65 \%$, entailing methicillin resistance in $77 \%$ of those cases. Less frequently, gram-negative bacteria were detected in $10 \%$ of our patient population. In consequence of our findings, calculated antibiotic treatment was changed to vancomycin and ceftriaxone in our institution.

With regard to time between primary/revision surgery and the number of needed revisions, no significant differences between low- and high-virulent pathogens were detected. However, both patients with Gaechter stage 3 were infected with high-virulent bacteria.

But considering the low number of included patients with Gaechter 3 and 4 arthritis, the impact of this finding remains unclear. Our results indicate that in a post-ACL reconstruction setting, low-virulent bacteria such as COSTs are able to induce acute joint infections with acute local and systemic signs as mentioned previously.

We did not administer antibiotics before arthroscopy but immediately afterward. Early onset of antibiotic treatment seems to play an important role in cartilage protection as animal model results suggest a time slot of 12 hours between onset of symptoms and first antibiotic application to minimize cartilage damage. ${ }^{13}$ But use of antibiotic agents before samples for microbiological evaluation were taken may impair bacterial identification and therefore targeted therapy in the setting of ACL infection.

In this study, the most commonly used antibiotic agents were quinolones, a substance class that is bactericidal and offers a good soft tissue penetration. ${ }^{19-21}$ The length of antibiotic therapy is controversial and ranges from 5 to 90 days. ${ }^{7,19}$ With 6 weeks of antibiotic treatment, we chose a relatively long administration period. Recently, approaches for a more individualized duration of antibiotic treatment based on CRP level were made, revealing a mean treatment period of $5.4 \pm 2.3$ weeks, ${ }^{16}$ which is very close to our interval.

Our study has various limitations. Besides the retrospective design and the rather short observational period, we did not perform a consideration of the outcome with a validated score.

Furthermore, inflammatory host response plays an important role in joint damage during septic arthritis. ${ }^{22}$ Once a joint infection has been established, there are two main mechanisms of infectious tissue damage, especially cartilage damage. One is the secretion of toxins by the pathogens itself. Staphylococci are the most commonly involved bacteria after ACL reconstruction ${ }^{17}$ and are known for toxin production. ${ }^{12}$ But the main reason for cartilage destruction seems to be the inflammatory host response to infection. ${ }^{22}$ Thus, the use of NSAIDs, which all patients received in our study and which reduce the inflammatory 
response, may have had a positive impact on the outcome, but it was not systematically considered in our work.

With $95 \%$ successful primary and $100 \%$ secondary infection treatment, and full range of motion and joint stability in $88 \%$ of the cases, the outcome in our cohort is rather good. However, it has to be taken into consideration that mainly patients with Gaechter stage 1 and 2 were treated. As is known, outcomes for Gaechter stage 3 and 4 are usually worse. $^{23}$ This is in line with our findings because the only case in which graft removal was necessary was a Gaechter stage 3 arthritis. Furthermore, the most frequent pathogens were COSTs, but the treatment of $S$. aureus might have been less successful. ${ }^{24,25}$

Another question is if our treatment concept is too aggressive. We performed at least three arthroscopic I\&D $(3.8 \pm 1.4)$, and the duration of antibiotic treatment was 6 weeks. It is unclear whether we would have been able to achieve the same results with fewer operations. Further research is needed to work out benchmarks and thresholds. Literature reveals the performance of 2.4 arthroscopic procedures before infection was cleared, ${ }^{1}$ though infections with $S$. aureus and age $<25$ years are associated risk factors for a higher number of operations during post-ACL reconstruction infection treatment. ${ }^{12}$ Thus, one interesting question for future observations is whether we need age- and pathogen-adapted treatment concepts in this entity. Another group achieved primary eradication of pathogens in $97 \%$ of 36 patients with 2.25 ( \pm 1.22 ) arthroscopic surgical procedures and an average antibiotic treatment period of 5.4 $( \pm 2.3)$ weeks. $^{16}$ In their setting, S. aureus was isolated in seven patients (21.9\%), and additionally, Enterobacter cloacae were detected in one case (3.1\%). Thus, good results are presented despite a relatively high proportion of aggressive bacteria, and, although not differing substantially, fewer surgeries and a shorter duration of antibiotic therapy were needed. Therapy was adjusted individually based on clinical findings and biomarker levels (CRP). This approach mandatorily requires closely monitored patients, which may not always be possible especially in outpatients.

Recently, another study that analyzed 30 patients with septic arthritis after ACL reconstruction was published and reported a $100 \%$ cure rate with arthroscopic debridement and an individualized antibiotic therapy, which led to a broad range of treatment duration (14-78 days; median: 23.5 days). ${ }^{18}$ Staphylococcus aureus was isolated in 12 (40\%) and gram-negative bacilli in 2 (7\%) patients. Nevertheless, results after I\&D and antibiotic therapy are highly variable, as pointed out by a systematic review that included 11 case series. ${ }^{17}$ Cure rates between 0 and $100 \%$ are published. Overall success rates for septic arthritis was $85.5 \%$, and average number of I\&D was 1.51 (range: $1-4) .{ }^{17}$ Again, the number of I\&Ds was determined based on clinical and laboratory findings, and the practice of routine repeated I\&D was not evident in the included studies. Multiple I\&Ds were necessary in $34.5 \%$ of patients. Clear criteria when I\&Ds have to be repeated are not defined, and the decision-making depends on the skills and experience of the surgeon. General risks and the required resources of an additional surgery must be properly weighed against the risk of persistent infection and cartilage damage in every case.

In this study, we present an effective, easy-to-perform, and safe standard treatment protocol for a rare complication, which may be helpful especially for less experienced centers.

\section{Conclusions}

In our study population, knee joint infection after ACL reconstruction presented as an acute event with local and systemic symptoms. Analysis of synovial fluid in blood culture bottles was a useful diagnostic tool. Beta-lactam resistant COSTs were the predominantly isolated pathogens. The most commonly used antibiotics were quinolones. Following our standard protocol consisting of at least three subsequent arthroscopic I\&Ds and 6 weeks of targeted antibiotic therapy, primary successful infection treatment with graft preservation was possible in 37 of the included 41 patients (90\%). Graft preservation was achieved in $100 \%$ of the included patients with Gaechter stage 1 and 2 infections.

\section{Conflict of Interest}

Author RO was paid for a scientific work for Pfizer Pharma $\mathrm{GmbH}$. Two included patients received Linezolid, which was exclusively distributed by Pfizer during the study period. The other authors declare that they have no conflict of interest.

\section{References}

1 Judd D, Bottoni C, Kim D, Burke M, Hooker S. Infections following arthroscopic anterior cruciate ligament reconstruction. Arthroscopy 2006;22(04):375-384

2 Scully WF, Fisher SG, Parada SA, Arrington ED. Septic arthritis following anterior cruciate ligament reconstruction: a comprehensive review of the literature. J Surg Orthop Adv 2013;22(02): 127-133

3 Williams RJ III, Laurencin CT, Warren RF, Speciale AC, Brause BD, O'Brien S. Septic arthritis after arthroscopic anterior cruciate ligament reconstruction. Diagnosis and management. Am J Sports Med 1997;25(02):261-267

4 Fong SY, Tan JL. Septic arthritis after arthroscopic anterior cruciate ligament reconstruction. Ann Acad Med Singapore 2004;33 (02):228-234

5 Parada SA, Grassbaugh JA, Devine JG, Arrington ED. Instrumentation-specific infection after anterior cruciate ligament reconstruction. Sports Health 2009;1(06):481-485

6 Schollin-Borg M, Michaëlsson K, Rahme H. Presentation, outcome, and cause of septic arthritis after anterior cruciate ligament reconstruction: a case control study. Arthroscopy 2003;19(09): 941-947

7 Kim S-J, Postigo R, Koo S, Kim JH. Infection after arthroscopic anterior cruciate ligament reconstruction. Orthopedics 2014;37 (07):477-484

8 Stutz G, Gächter A. Diagnostik und stadiengerechte Therapie von Gelenkinfekten. Unfallchirurg 2001;104(08):682-686

9 Centers for Disease Control and Prevention. CDC/NHSN Surveillance Definitions for Specific Types of Infections. Available at: https://www.cdc.gov/nhsn/PDFs/pscManual/17pscNosInfDef_current.pdf

10 Kuršumović K, Charalambous CP. Graft salvage following infected anterior cruciate ligament reconstruction: a systematic review and meta-analysis. Bone Joint J 2016;98-B(05):608-615 
11 Calvisi V, Lupparelli S. C-reactive protein changes in the uncomplicated course of arthroscopic anterior cruciate ligament reconstruction. Int J Immunopathol Pharmacol 2008;21(03):603-607

12 Mouzopoulos G, Fotopoulos VC, Tzurbakis M. Septic knee arthritis following ACL reconstruction: a systematic review. Knee Surg Sports Traumatol Arthrosc 2009;17(09):1033-1042

13 Wang C, Ao Y, Fan X, et al. changes after arthroscopic anterior cruciate ligament postoperative infection. Arthrosc J Arthrosc Relat Surg 2014;30(09):1110-1115

14 Wang C, Ao Y, Fan X, et al. C-reactive protein and erythrocyte sedimentation rate changes after arthroscopic anterior cruciate ligament reconstruction: guideline to diagnose and monitor postoperative infection. Arthrosc J Arthrosc. Relat Surg 2014;30 (09):1110-1115

15 Trampuz A, Piper KE, Jacobson MJ, et al. Sonication of removed hip and knee prostheses for diagnosis of infection. N Engl J Med 2007; 357(07):654-663

16 Schuster P, Schulz M, Immendoerfer M, Mayer P, Schlumberger M, Richter J. Septic arthritis after arthroscopic anterior cruciate ligament reconstruction: evaluation of an arthroscopic graft-retaining treatment protocol. Am J Sports Med 2015;43(12):3005-3012

17 Saper M, Stephenson K, Heisey M. Arthroscopic irrigation and debridement in the treatment of septic arthritis after anterior cruciate ligament reconstruction. Arthroscopy 2014;30(06):747-754

18 Erice A, Neira MI, Vargas-Prada S, Chiaraviglio A, GutiérrezGuisado J, Rodríguez de Oya R. Septic arthritis following arthro- scopic reconstruction of cruciate ligaments of the knee: retrospective case review. Enferm Infecc Microbiol Clin 2018;36(06): 336-341

19 Bellmann R, Kuchling G, Dehghanyar P, et al. Tissue pharmacokinetics of levofloxacin in human soft tissue infections. Br J Clin Pharmacol 2004;57(05):563-568

20 Landersdorfer CB, Bulitta JB, Kinzig M, Holzgrabe U, Sörgel F. Penetration of antibacterials into bone: pharmacokinetic, pharmacodynamic and bioanalytical considerations. Clin Pharmacokinet 2009;48(02):89-124

21 Joukhadar C, Stass H, Müller-Zellenberg U, et al. Penetration of moxifloxacin into healthy and inflamed subcutaneous adipose tissues in humans. Antimicrob Agents Chemother 2003;47(10): 3099-3103

22 Goldenberg DL. Septic arthritis. Lancet 1998;351(9097):197-202

23 Petersen W, Herbort M, Höynck E, Zantop T, Mayr H. Stageadapted treatment of infection after reconstruction of the anterior cruciate ligament . [in German]. Oper Orthop Traumatol 2014;26(01):63-74

24 Calvo R, Figueroa D, Anastasiadis Z, et al. Septic arthritis in ACL reconstruction surgery with hamstring autografts. Eleven years of experience. Knee 2014;21(03):717-720

25 Maletis GB, Inacio MCS, Reynolds S, Desmond JL, Maletis MM, Funahashi TT. Incidence of postoperative anterior cruciate ligament reconstruction infections: graft choice makes a difference. Am J Sports Med 2013;41(08):1780-1785 